\title{
Carboxylated Capped Carbon Nanotubes Interacting with Nimesulide Molecules: Applied Electric Fields Effects
}

\author{
Vivian Machado de Menezes, ${ }^{1}$ Ivana Zanella, ${ }^{2}$ and Solange Binotto Fagan ${ }^{2}$ \\ ${ }^{1}$ Universidade Federal da Fronteira Sul (UFFS), Campus Laranjeiras do Sul, 85301-970 Laranjeiras do Sul, PR, Brazil \\ ${ }^{2}$ Pós-Graduação em Nanociências, Centro Universitário Franciscano (UNIFRA), 97010-032 Santa Maria, RS, Brazil \\ Correspondence should be addressed to Vivian Machado de Menezes; demenezes.vivian@gmail.com
}

Received 20 August 2015; Accepted 2 November 2015

Academic Editor: Yuan Chen

Copyright (c) 2015 Vivian Machado de Menezes et al. This is an open access article distributed under the Creative Commons Attribution License, which permits unrestricted use, distribution, and reproduction in any medium, provided the original work is properly cited.

\begin{abstract}
Interactions of carboxylated capped carbon nanotubes with nimesulide molecules under electric fields were investigated by ab initio simulations. Repulsive forces between the nimesulide molecules and the carboxyl group of the carbon nanotubes, except for the nimesulide radical configuration, were observed. To keep the original molecule in the pristine form, electric fields with different intensities were applied, where changes in the behavior of the interactions between the molecules were noticed. It was shown that the intensity of the interaction between the nimesulide and the hydrophilic carboxylated capped carbon nanotube can be modulated by the action of the external electric fields making promising systems for drug delivery applications.
\end{abstract}

\section{Introduction}

In the world of nanotechnology, carbon nanotube $(\mathrm{CN})$ represents one of the most promising materials for applications in several knowledge areas, because of its exceptional electronic, mechanical, and thermal properties [1]. Due to their susceptibility to chemical interactions, CNs are very favorable for biomedical applications; besides they have dimensions comparable to many biological species.

As nanomaterial for biomedical applications, the use of $\mathrm{CN}$ includes biological sensors [1] and drug delivery systems [2], among other possibilities [3]. Until now, it is known that pristine $\mathrm{CN}$ presents certain toxicity, but the functionalized one can overcome this problem and could present good biocompatibility, which is crucial for applying this material in biomedical area [4]. Another point to take into account for the successful application of nanotubes in biomedical devices is the fact that, in pristine form, they are hydrophobic and biological fluids are aqueous [3]. It is reported that the introduction of functional groups on the tube surface can surpass these difficulties, and the carboxyl group is the most used one for this goal [5]. In addition to the functionalization of CNs, some interactions may be facilitated by the application of electric fields, which polarizes the system inducing a dipolar momentum, changing the nonpolar character of the tube [6].

Nimesulide is a nonsteroidal anti-inflammatory drug belonging to the class of sulfonamides with anti-inflammatory, antipyretic, and analgesic effect. Despite a wide range of applications, the nimesulide may cause some gastrointestinal disorders [7]. In toxicity studies, it presents essentially liver, renal, and gastrointestinal damage. In Switzerland [8], based on the number of cases reported, it was considered the most hepatotoxic anti-inflammatory drug. Due to studies like this, the use of nimesulide was discontinued in some countries, such as Ireland and many other European countries [9]. Moreover, nimesulide can cause other effects when in higher dose than therapeutic concentrations, which can occur in tissues where the drug is adsorbed, for example, antioxidant activity. The direct delivery of nimesulide molecules to specific tissues could maximize and enhance the positive effects of the drug on the human body as well as avoid the undesirable side effects. The possibility here proposed is the interaction of nimesulide molecules with carboxylated capped carbon nanotubes (CCCN). These nanomaterials can provide an alternative route of drug delivery to a specific part of the body and the carboxyl group may represent a way to control the insolubility and incompatibility of CN [10]. 
The interactions of nimesulide with pristine and $\mathrm{Si}$-doped capped $\mathrm{CN}$ were previously studied by Zanella et al. [11] through first principles calculations, where a physisorption regime was observed. In this paper, repulsive forces were found in the studied interactions, and we proposed overcoming the drawbacks by the application of an electric field, which changes the binding energy values. Previous studies show that the applied electric field can polarize the system, increasing the interactions of $\mathrm{CN}$ with polar molecules or even allowing covalent bonds between these systems $[6,12,13]$.

\section{Methodology}

The structural and energetic results for CCCN interacting with nimesulide were performed by $a b$ initio density functional theory (DFT) calculations $[14,15]$. The SIESTA code [16] was used to execute the simulations and interactions between ionic cores and valence electrons using normconserving pseudopotentials (Troullier-Martins) [17]. The double-zeta basis set plus polarization functions (DZP) were employed for all atomic species. The exchange-correlation potential was adjusted by the local density approximation (LDA), according to the parameterization proposed by Perdew and Zunger [18]. A cutoff of $200 \mathrm{Ry}$ for the grid integration in the real space was used, to represent the charge density. The structural optimizations were achieved through the conjugate gradient algorithm [16] until the residual forces were smaller than $0.05 \mathrm{eV} / \AA$.

The binding energies $\left(E_{b}\right)$ were calculated using the basis set superposition error (BSSE) [19]. This correction is done through the counterpoise method using "ghost" atoms within the following equation:

$$
\begin{aligned}
E_{b} & =\left[E_{T}(\mathrm{CCCN}+\mathrm{X})-E_{T}\left(\mathrm{CCCN}+\mathrm{X}_{\text {ghost }}\right)\right. \\
& \left.-E_{T}\left(\mathrm{CCCN}_{\text {ghost }}+\mathrm{X}\right)\right]
\end{aligned}
$$

where $\mathrm{X}$ corresponds to the nimesulide molecule and $E_{T}(\mathrm{CCCN}+\mathrm{X})$ is the total energy of CCCN interacting with nimesulide. The "ghost" CCCN/X corresponds to additional basis wave functions centered at the position of the CCCN/X but without any atomic potential.

In order to change the interactions between nimesulide molecules and CCCN, electric fields with different intensities were applied, with the same criteria as studied by de Menezes et al. [6] that considered values of electric field within a range considered practicable not to promote the breakage of the carbon bonds of the nanotubes. The relative energy $\left(E_{r}\right)$ was calculated as the difference between the total energies of the system with/without the applied electric field $\left(E_{E}\right) /\left(E_{0}\right)$ :

$$
E_{r}=E_{E}-E_{0}
$$

All the calculations were performed using periodicboundary conditions and supercell approximations. A lateral separation of $40 \AA$ between tube centers was used in order to guarantee that there was no interaction among the periodic images in the perpendicular direction to the tube axis. The unity cell had 120 carbon atoms plus the carboxyl radical,
TABLE 1: Distances and binding energies for the configurations of Figure 1.

\begin{tabular}{lcc}
\hline System & Bond distance $(\AA)$ & $E_{b}(\mathrm{eV})$ \\
\hline (i) & $1.99\left(\mathrm{O}-\mathrm{O}_{\text {tube }}\right)$ & 2.09 \\
(ii) & $2.15\left(\mathrm{O}-\mathrm{O}_{\text {tube }}\right)$ & 0.62 \\
(iii) & $1.36\left(\mathrm{C}-\mathrm{O}_{\text {tube }}\right)$ & -3.85 \\
\hline
\end{tabular}

and the tube was $(10,0)$, with a diameter suitable for closing the tube ends with fragments of fullerene $\mathrm{C}_{60}$. All geometry optimizations were done without any symmetry constraints.

\section{Results}

Three configurations were analyzed of the nimesulide moving toward the carboxyl group of the CCCN by the following: (i) oxygen of the $-\mathrm{NO}_{2}$ group (same configuration studied by Zanella et al. [11], Figure 1(c)), labeled nime-N; (ii) the $-\mathrm{SO}_{2}$ group (Figure $1(\mathrm{~d})$ ), labeled nime-S; and (iii) the $-\mathrm{CH}_{2}$ nimesulide radical (removing one hydrogen from the $-\mathrm{CH}_{3}$ group) (Figure 1(e)).

Table 1 shows the values of binding energy (calculated by (1)) and smaller distance between both molecules (CCCN and nimesulide), for the considered configurations. One can observe that the interaction between the oxygen of the nimesulide and the carboxyl group of the tube tends to be repulsive, resulting in a positive binding energy.

Figure 2 shows the energy levels and the plot of the local density of states (LDOS) for all systems of Figure 1. For pristine nimesulide (Figure 2(a)), the plot of electronic charge densities for HOMO (highest occupied molecular orbital) and LUMO (lowest unoccupied molecular orbital) indicates that there is no contribution of the charge from $-\mathrm{CH}_{3}$ group. That indicates that this group in the original form is the less reactive part of nimesulide, and to change this scenario, we functionalized the nimesulide molecule removing one hydrogen from this group, forming a dangling bond. For the configuration of the interacting systems in which the $-\mathrm{CH}_{3}$ group is functionalized by removing one hydrogen, a covalent bond is observed. In this configuration, the HOMO and LUMO energy difference $(\Delta \mathrm{HL})$ is almost closed (Figure 2(e)). From the plot of LDOS, as shown in Figure 2(e), a small contribution of the charge from the region of the interaction in the $\mathrm{HOMO}$ and a major contribution of this region in the LUMO are observed. In HOMO, the major contribution is in the opposite side to the interaction region, as in the CCCN without the nimesulide (Figure 2(b)). In the nime- $\mathrm{N}$ configuration, a charge contribution occurs mainly from the interaction region in the HOMO (Figure 2(c)).

Considering the fact that nimesulide interacts only covalently when functionalized, we apply an external uniform electric field in the CCCN axis direction in order to modulate the intensity of the interaction in the nime$\mathrm{N}$ and nime-S arrangements. For a drug delivery system, a physisorption regime is appropriate due to the possibility of attaching/detaching molecules from a nanostructure, and a covalent bond is too strong to allow this mechanism. Because of that, configuration (iii) (functionalized nimesulide) is 


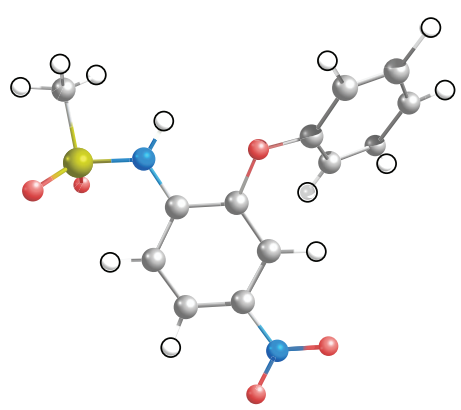

(a)

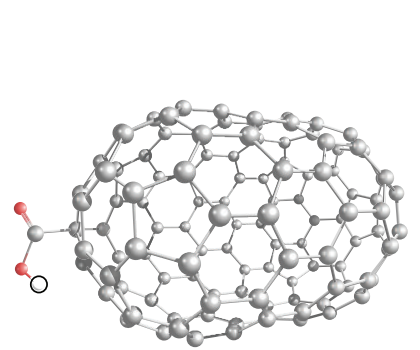

(b)

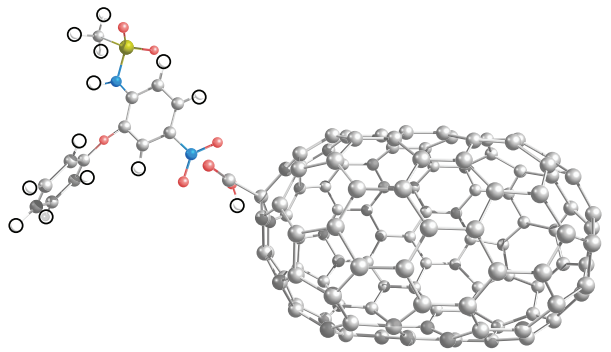

(c)

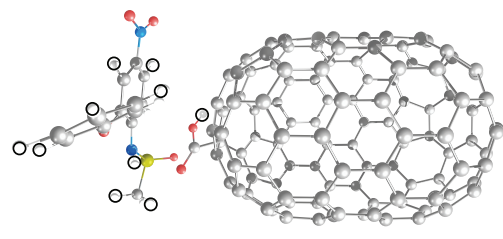

(d)

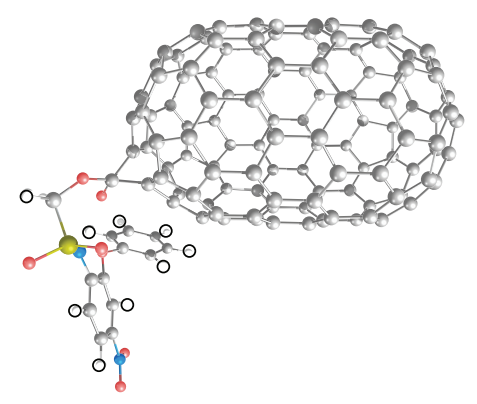

(e)

FIGURE 1: Optimized geometry of (a) isolated nimesulide, (b) isolated CCCN, (c) nime-N, (d) nime-S, and (e) functionalized nimesulide interacting with the nanotube by the $-\mathrm{CH}_{2}$ group. Grey: carbon; white: hydrogen; blue: nitrogen; red: oxygen; and yellow: sulfur.

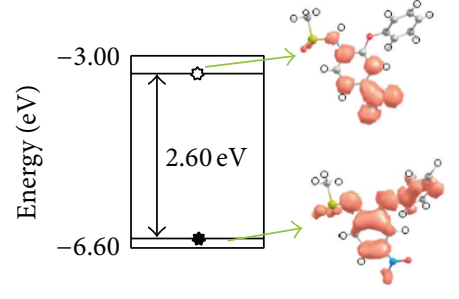

(a)

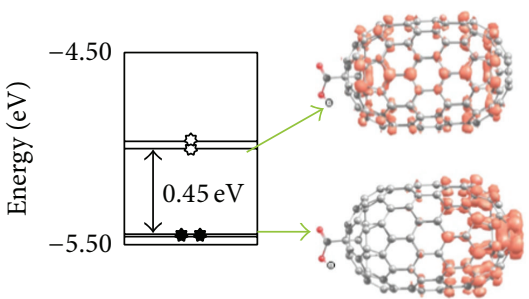

(b)

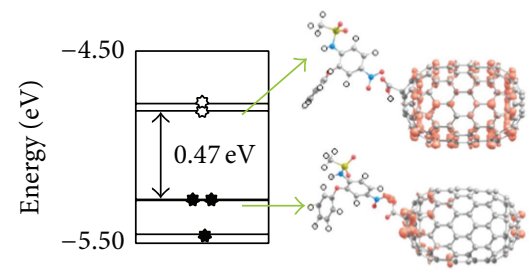

(c)

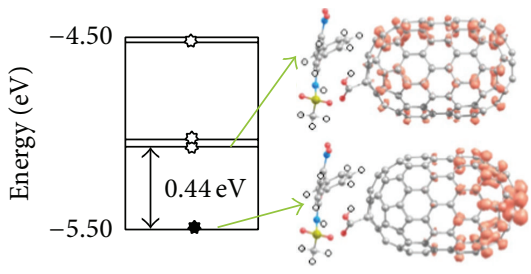

(d)

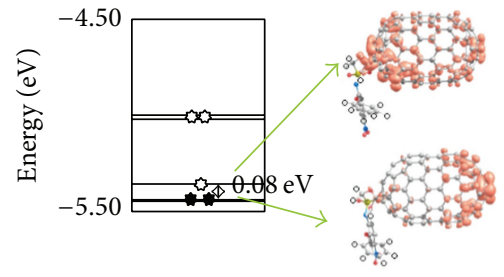

(e)

FIGURE 2: Energy levels and plots of the electronic charge densities (LDOS) for HOMO and LUMO for (a) isolated nimesulide, (b) isolated CCCN, (c) nime-N, (d) nime-S, and (e) nimesulide radical interacting with the CCCN by the $-\mathrm{CH}_{2}$ group. Filled circles are valence electrons and empty circles are conduction electrons. The isodensity used for the charge density plots was $0.0005 \mathrm{e} / \mathrm{Bohr}^{3}$.

not considered for the application of the external electric field. The scheme of Figure 3 illustrates how electric fields of $0.05 \mathrm{~V} / \AA$ were applied on the systems with the isolated nimesulide; similar methodology was previously used by de Menezes et al. [6]. It is relevant to point out that no spin polarization was observed for the most stable configurations.

Different intensities and directions of the applied electric field $(-0.05,0$, and $0.05 \mathrm{~V} / \AA)$ were checked for the configurations nime- $\mathrm{N}$ and nime-S. The results for the relative energy (see (2)) show that the stability of the systems occurs with applied electric field parallel [antiparallel] to the tube axis for nime- $\mathrm{N}$ [nime-S].

In this way, we consider for nime-N [nime-S] high intensities of the applied electric fields in parallel [antiparallel] direction to the nanotube axis, with the results presented in Table 2. It is not surprising that different configuration requires field applied on opposite orientations. Similar behavior was described by de Menezes et al. [6] when which 


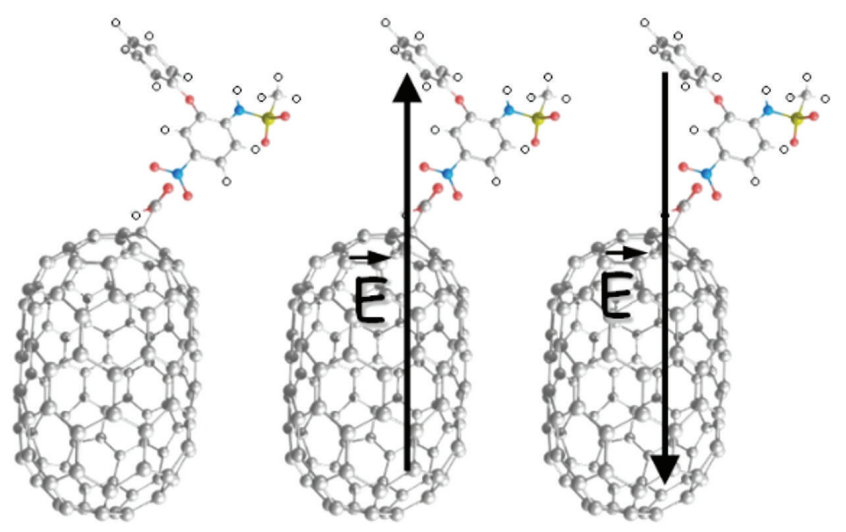

(a)
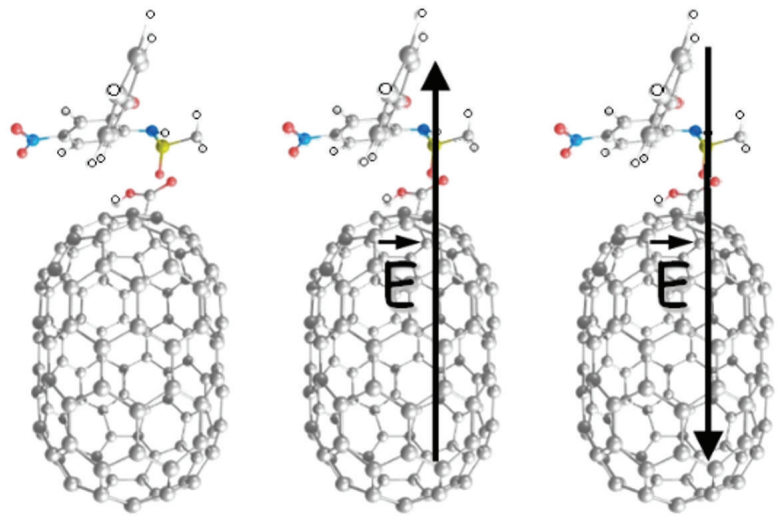

(b)

FIGURE 3: Illustration of the electric field applied to the systems (a) nime- $\mathrm{N}$ and (b) nime-S configurations.

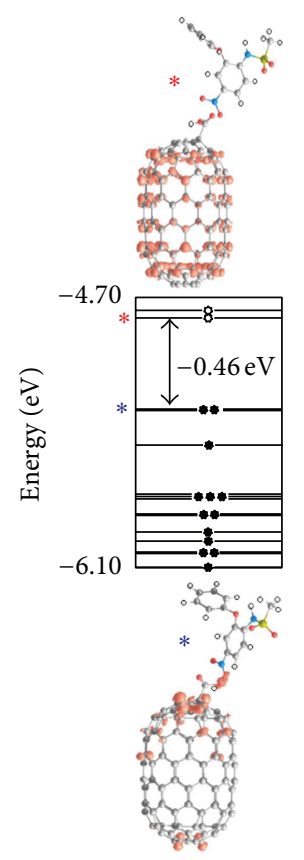

(a)

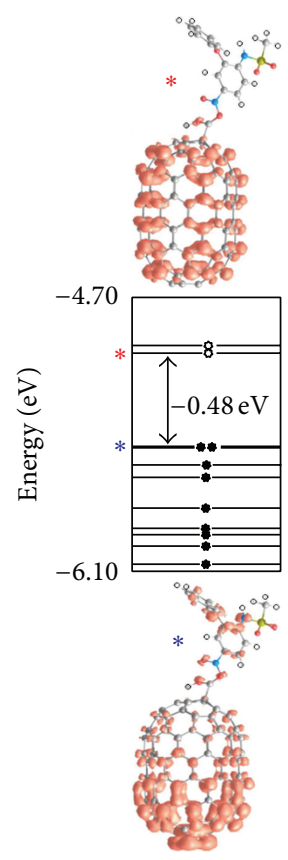

(b)

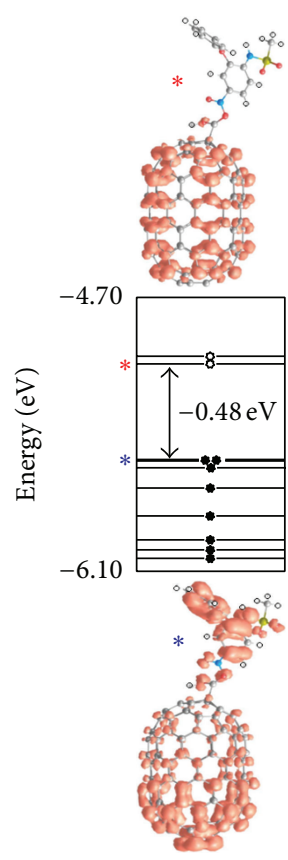

(c)

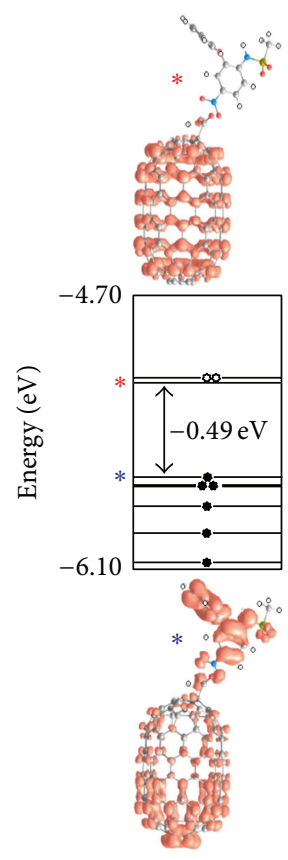

(d)

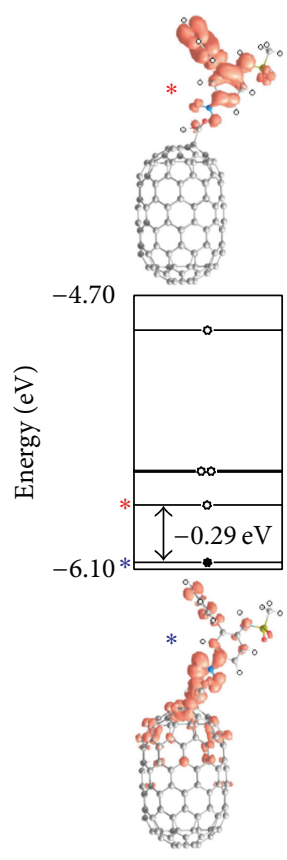

(e)

FIGURE 4: Energy levels and plots of the electronic charge densities for HOMO (assigned by the blue stars) and LUMO (red stars) for the nime-N system under the electric fields of (a) 0 , (b) 0.05 , (c) 0.10 , (d) 0.20 , and (e) $0.40 \mathrm{~V} / \AA$. For the LDOS plots, the isodensity used was of $0.0005 \mathrm{e} / \mathrm{Bohr}^{3}$ for all the cases and the energy levels ranges were between -6.10 and $-4.70 \mathrm{eV}$.

orientation of electric field was more favorable to stabilize CN functionalized by amine, amide, hydroxyl, and carboxyl was being determined.

For the nime- $\mathrm{N}$ a small rise in the $\Delta \mathrm{HL}$ of the systems until the electric field is $0.20 \mathrm{~V} / \AA$ is observed, with an increase in the instability of the systems, as can be observed on the relative binding energies. In case of the nime-S configuration, for all values for the applied electric fields, a reduction on the $\Delta \mathrm{HL}$ and a growth in the energetic stability are observed.

Figure 4 presents the energy levels and the corresponding LDOS plots for the nime-N configuration with different intensities of applied electric field. A significant reduction of $\Delta \mathrm{HL}$ around $0.40 \mathrm{~V} / \AA$ is also observed. In general, the modification on the electronic charge distribution for the HOMO and LUMO levels is observed due to the tendency to polarize the resulting systems. In the specific case of the nime- $\mathrm{N}$ under electric field of $0.40 \mathrm{~V} / \AA$, the electronic charge in the LUMO moves to nimesulide molecule, mainly in the opposite region of the interaction. In the same case, the electronic charge for the HOMO is located in the region of the interaction between the CCCN and the nimesulide.

For the nime-S, as can be seen in Figure 5, the action of the electric field with intensity of $-0.40 \mathrm{~V} / \AA$ presents the same trend observed for the charge distribution in the LUMO level for the nime-N system. The HOMO level presents 


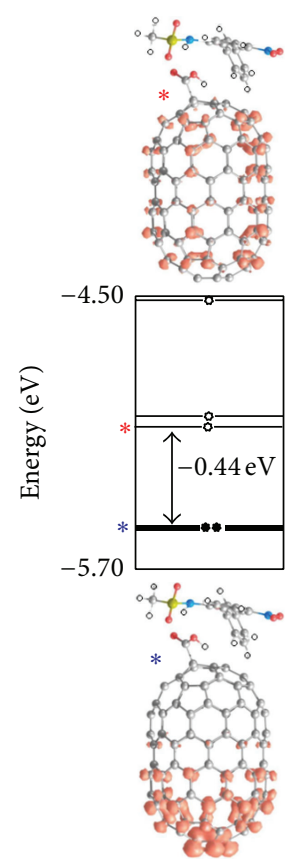

(a)

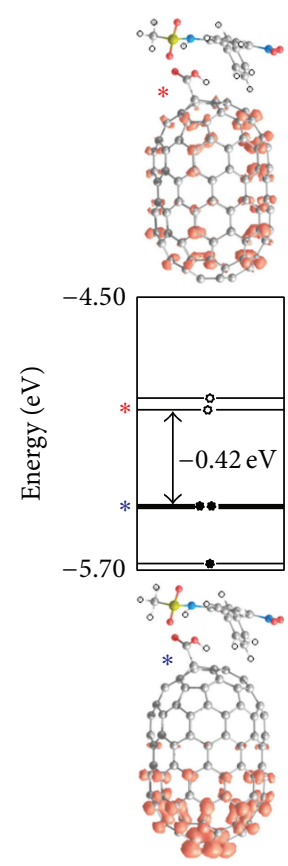

(b)

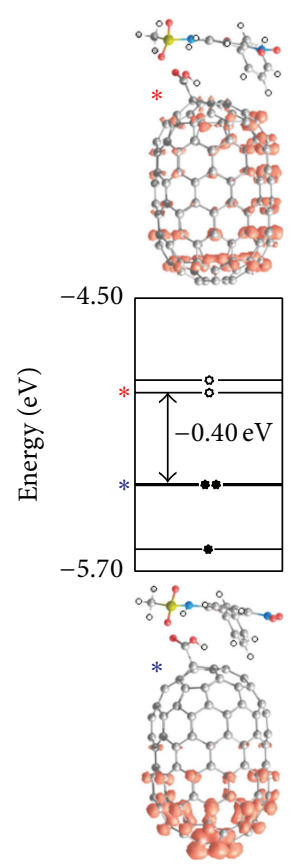

(c)

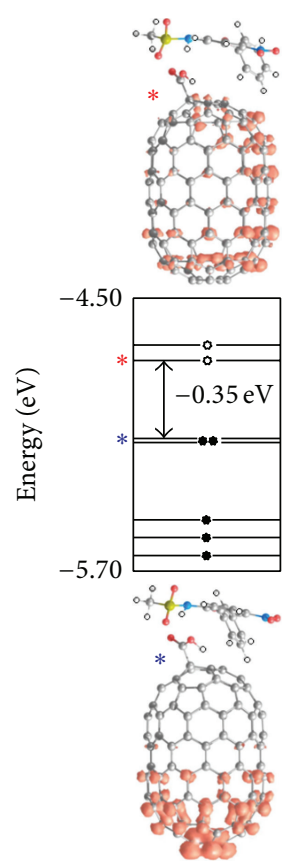

(d)

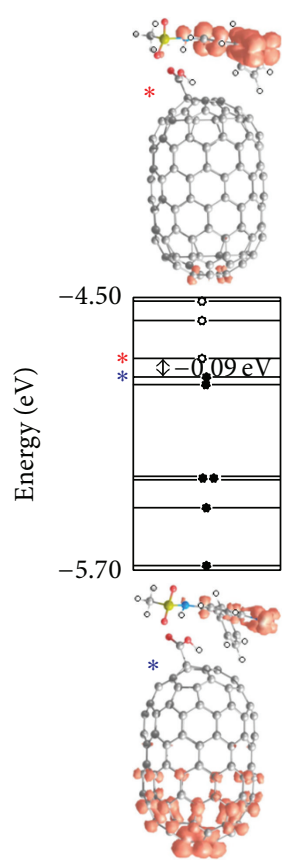

(e)

FIGURE 5: Energy levels and plots of the electronic charge densities for HOMO (assigned by the blue stars) and LUMO (red stars) for the system nime-S under the action of electric fields of (a) 0 , (b) $-0.05 \mathrm{~V} / \AA$, (c) $-0.10 \mathrm{~V} / \AA$, (d) $-0.20 \mathrm{~V} / \AA$, and (e) $-0.40 \mathrm{~V} / \AA$. The isodensity was of $0.0005 \mathrm{e} / \mathrm{Bohr}^{3}$ for all the cases and the energy levels were between -5.70 and $-4.50 \mathrm{eV}$.

TABLE 2: Relative energies, binding energies, and $\triangle H L$ values for CCCN interacting with the systems nime-N and nime-S under the action of different values of applied electric fields.

\begin{tabular}{ccccc}
\hline & $\begin{array}{c}\text { Electric } \\
\text { field }(\mathrm{V} / \AA)\end{array}$ & $E_{r}(\mathrm{eV})$ & $E_{b}(\mathrm{eV})$ & $\Delta \mathrm{HL}(\mathrm{eV})$ \\
\hline \multirow{4}{*}{ Nime-N } & 0 & - & 2.09 & 0.46 \\
& 0.05 & -0.14 & 2.06 & 0.48 \\
& 0.10 & -0.41 & 1.94 & 0.48 \\
& 0.20 & -1.40 & 1.41 & 0.49 \\
& 0.40 & -5.38 & -1.02 & 0.29 \\
\hline \multirow{3}{*}{ Nime-S } & 0 & - & 0.62 & 0.44 \\
& -0.05 & -0.07 & 0.61 & 0.42 \\
& -0.10 & -0.20 & 0.58 & 0.40 \\
& -0.20 & -0.66 & 0.49 & 0.35 \\
& -0.40 & -2.34 & 0.19 & 0.09 \\
\hline
\end{tabular}

electronic charge in the opposite regions to the interaction, both in the nanotube and in the nimesulide molecule.

Besides, some gas adsorption behavior on carbon based materials has been investigated, where the application of an external electric field can modulate the adsorption/desorption of the molecules being able to act as a reversible switch $[20,21]$.

The explanation for enhancement (or even weakening) of these interactions is the induction of an extra dipole moment and the appearing of the Stark effect, which causes the spin splitting and degeneracy breaking of energy levels [6, 20, 21].
It is possible to see in [6] a systematic study of the polarization associated with the electric dipole moment of CCCN induced by electric fields of the same intensities here studied. The degeneracy breaking of energy levels in our study is more evident in HOMO levels in both cases (nime-N (Figure 4) and nime-S (Figure 5)), as well as in all cases of functionalized carbon nanotubes studied in [6]. The external electric field can also modulate the bonding properties of van der Waals complexes between $\pi$-systems and other species through the polarization of the systems $[22,23]$.

\section{Conclusions}

In summary, different arrangements for the nimesulide molecules and CCCN interactions were reported here. However, the trend is that the pristine nimesulide molecule does not present chemical bond with CCCN, these nanostructures being not favorable for carrying out nimesulide in the original form. In both studied configurations of pristine nimesulide interacting with CCCN positive values of binding energies were observed.

Nevertheless, considering carbon nanotubes as component of drug delivery systems, it would be interesting to show the physical interaction between these systems. Then, a way to get the physical adsorption modulated by applying external electric fields was proposed here. We have demonstrated that the interaction between the CCCN and the nimesulide molecule becomes more favorable when exposed to the action of an external electric field, and this knowledge could be extended to interactions with other molecules with 
nanotubes. It is important to observe that values out of the applied electric field range considered in this work can produce negative effects associated with break of chemical bonds of the original systems (carbon nanotubes and/or adsorbed molecules), creating structures with different properties that are not of interest for drug delivery.

Then, the studied systems are relevant for biomedical applications because they can combine the hydrophilic properties of the CCCN with the modulation of the intensity of the nimesulide interaction through external physical agents.

\section{Conflict of Interests}

The authors declare that there is no conflict of interests regarding the publication of this paper.

\section{Acknowledgments}

The authors acknowledge CENAPAD-SP for computer time and the Brazilian agencies CNPq and FAPERGS for financial support.

\section{References}

[1] K. K. Kamil Reza, S. Srivastava, S. K. Yadav, and A. M. Biradar, "Biofunctionalized carbon nanotubes platform for biomedical applications," Materials Letters, vol. 126, pp. 126-130, 2014.

[2] N. K. Mehra, V. Mishra, and N. K. Jain, "A review of ligand tethered surface engineered carbon nanotubes," Biomaterials, vol. 35, no. 4, pp. 1267-1283, 2014.

[3] S. Vardharajula, S. Z. Ali, P. M. Tiwari et al., "Functionalized carbon nanotubes: biomedical applications," International Journal of Nanomedicine, vol. 7, pp. 5361-5374, 2012.

[4] S. K. Smart, A. I. Cassady, G. Q. Lu, and D. J. Martin, "The biocompatibility of carbon nanotubes," Carbon, vol. 44, no. 6 , pp. 1034-1047, 2006.

[5] I. V. Lara, I. Zanella, A. G. de Souza Filho, and S. B. Fagan, "Influence of concentration and position of carboxyl groups on the electronic properties of single-walled carbon nanotubes," Physical Chemistry Chemical Physics, vol. 16, no. 39, pp. 2160221608, 2014.

[6] V. M. de Menezes, R. Mota, I. Zanella, and S. B. Fagan, "Pristine and functionalized capped carbon nanotubes under electric fields," Physica Status Solidi B: Basic Research, vol. 251, no. 3, pp. 649-654, 2014.

[7] J. P. Famaey, "In vitro and in vivo pharmacological evidence of selective cyclooxygenase- 2 inhibition by nimesulide: an overview," Inflammation Research, vol. 46, no. 11, pp. 437-446, 1997.

[8] G. Merlani, M. Fox, H.-P. Oehen et al., "Fatal hepatotoxicity secondary to nimesulide," European Journal of Clinical Pharmacology, vol. 57, no. 4, pp. 321-326, 2001.

[9] M. Venegoni, R. Da Cas, F. Menniti-Ippolito, and G. Traversa, "Effects of the European restrictive actions concerning nimesulide prescription: a simulation study on hepatopathies and gastrointestinal bleedings in Italy," Annali dell'Istituto Superiore di Sanità, vol. 46, no. 2, pp. 153-157, 2010.

[10] N. Mali, S. Jadhav, M. Karpe, and V. Kadam, "Carbon nanotubes as carriers for delivery of bioactive and therapeutic agents: an overview," International Journal of Pharmacy and Pharmaceutical Sciences, vol. 3, no. 3, pp. 45-52, 2011.

[11] I. Zanella, S. B. Fagan, R. Mota, and A. Fazzio, "Ab initio study of pristine and Si-doped capped carbon nanotubes interacting with nimesulide molecules," Chemical Physics Letters, vol. 439, no. 4-6, pp. 348-353, 2007.

[12] M. Grujicic, G. Cao, and B. Gersten, "Enhancement of field emission in carbon nanotubes through adsorption of polar molecules," Applied Surface Science, vol. 206, no. 1-4, pp. 167$177,2003$.

[13] A. Maiti, J. Andzelm, N. Tanpipat, and P. von Allmen, "Effect of adsorbates on field emission from carbon nanotubes," Physical Review Letters, vol. 87, no. 15, Article ID 155502, 2001.

[14] P. Hohenberg and W. Kohn, "Inhomogeneous electron gas," Physical Review B, vol. 136, pp. 864-871, 1964.

[15] W. Kohn and L. J. Sham, "Self-consistent equations including exchange and correlation effects," Physical Review A, vol. 140, no. 4, pp. A1133-A1138, 1965.

[16] J. M. Soler, E. Artacho, J. D. Gale et al., "The SIESTA method for ab initio order-N materials simulation," Journal of Physics: Condensed Matter, vol. 14, no. 11, pp. 2745-2779, 2002.

[17] N. Troullier and J. L. Martins, "Efficient pseudopotentials for plane-wave calculations," Physical Review B, vol. 43, no. 3, pp. 1993-2006, 1991.

[18] J. P. Perdew and A. Zunger, "Self-interaction correction to density-functional approximations for many-electron systems," Physical Review B, vol. 23, no. 10, pp. 5048-5079, 1981.

[19] S. Boys and F. Bernardi, "The calculation of small molecular interactions by the differences of separate total energies. Some procedures with reduced errors," Molecular Physics, vol. 19, no. 4, pp. 553-566, 2006.

[20] Z. M. Ao, S. Li, and Q. Jiang, "Correlation of the applied electrical field and $\mathrm{CO}$ adsorption/desorption behavior on Aldoped graphene," Solid State Communications, vol. 150, no. 1314, pp. 680-683, 2010.

[21] W. Liu, Y. H. Zhao, Y. Li, E. J. Lavernia, and Q. Jiang, "A reversible switch for hydrogen adsorption and desorption: electric fields," Physical Chemistry Chemical Physics, vol. 11, no. 40, pp. 9233-9240, 2009.

[22] C. Foroutan-Nejad and R. Marek, "Potential energy surface and binding energy in the presence of an external electric field: modulation of anion- $\pi$ interactions for graphene-based receptors," Physical Chemistry Chemical Physics, vol. 16, no. 6, pp. 2508-2514, 2014.

[23] G. C. Loh, S. Nigam, G. Mallick, and R. Pandey, "Carbon-doped boron nitride nanomesh: stability and electronic properties of adsorbed hydrogen and oxygen," Journal of Physical Chemistry C, vol. 118, no. 41, pp. 23888-23896, 2014. 

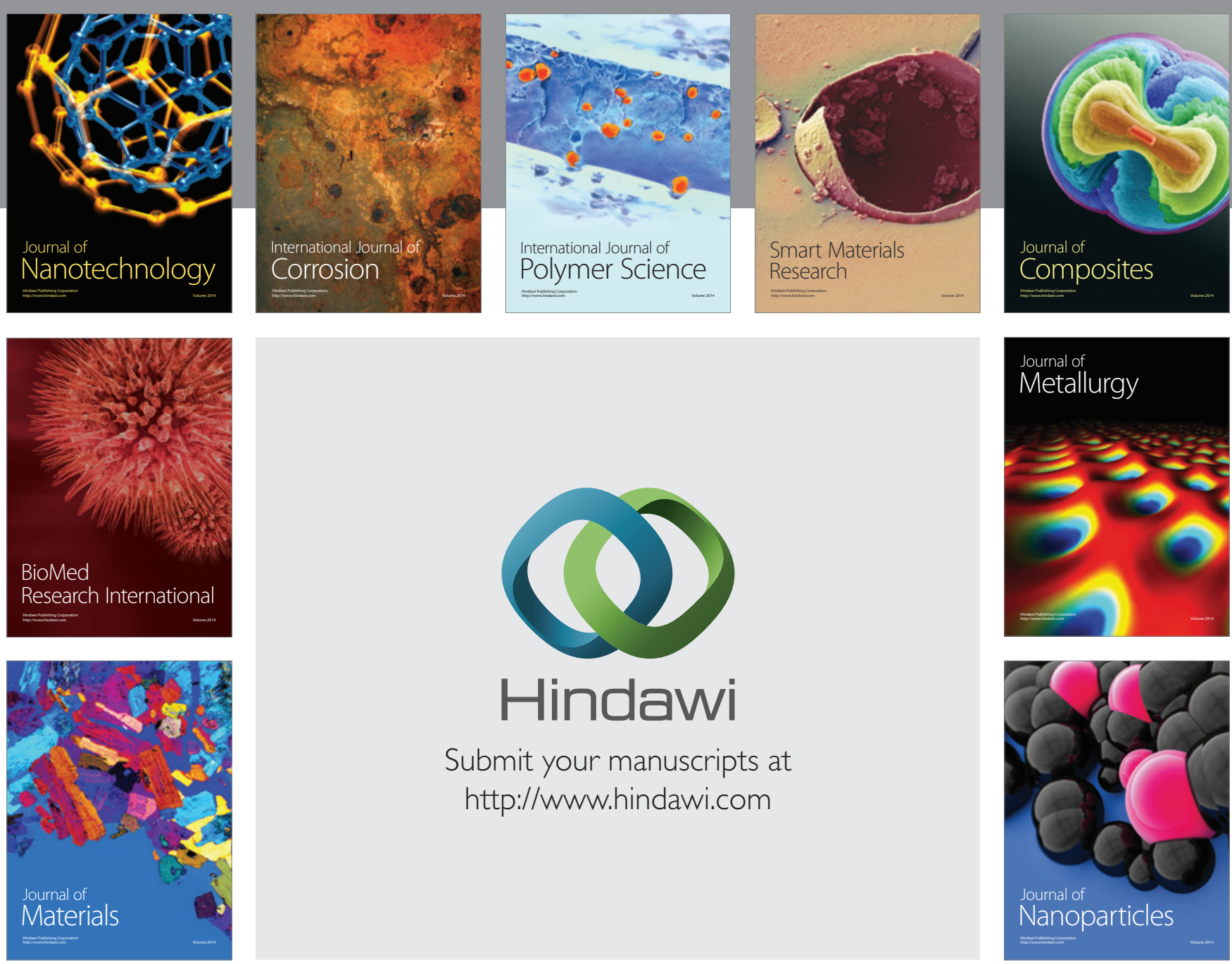

Submit your manuscripts at http://www.hindawi.com
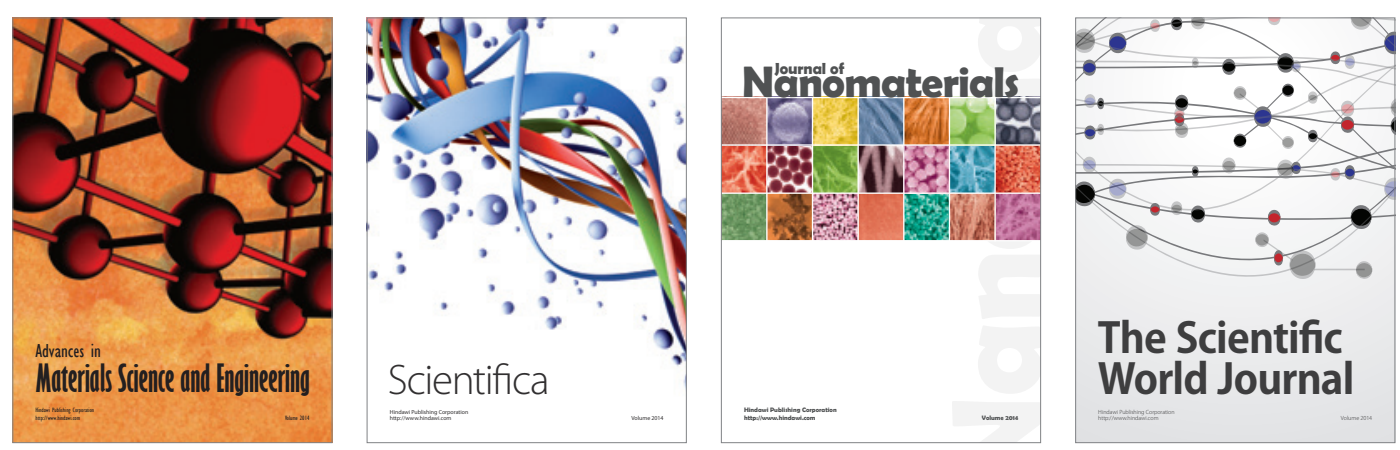

\section{The Scientific World Journal}
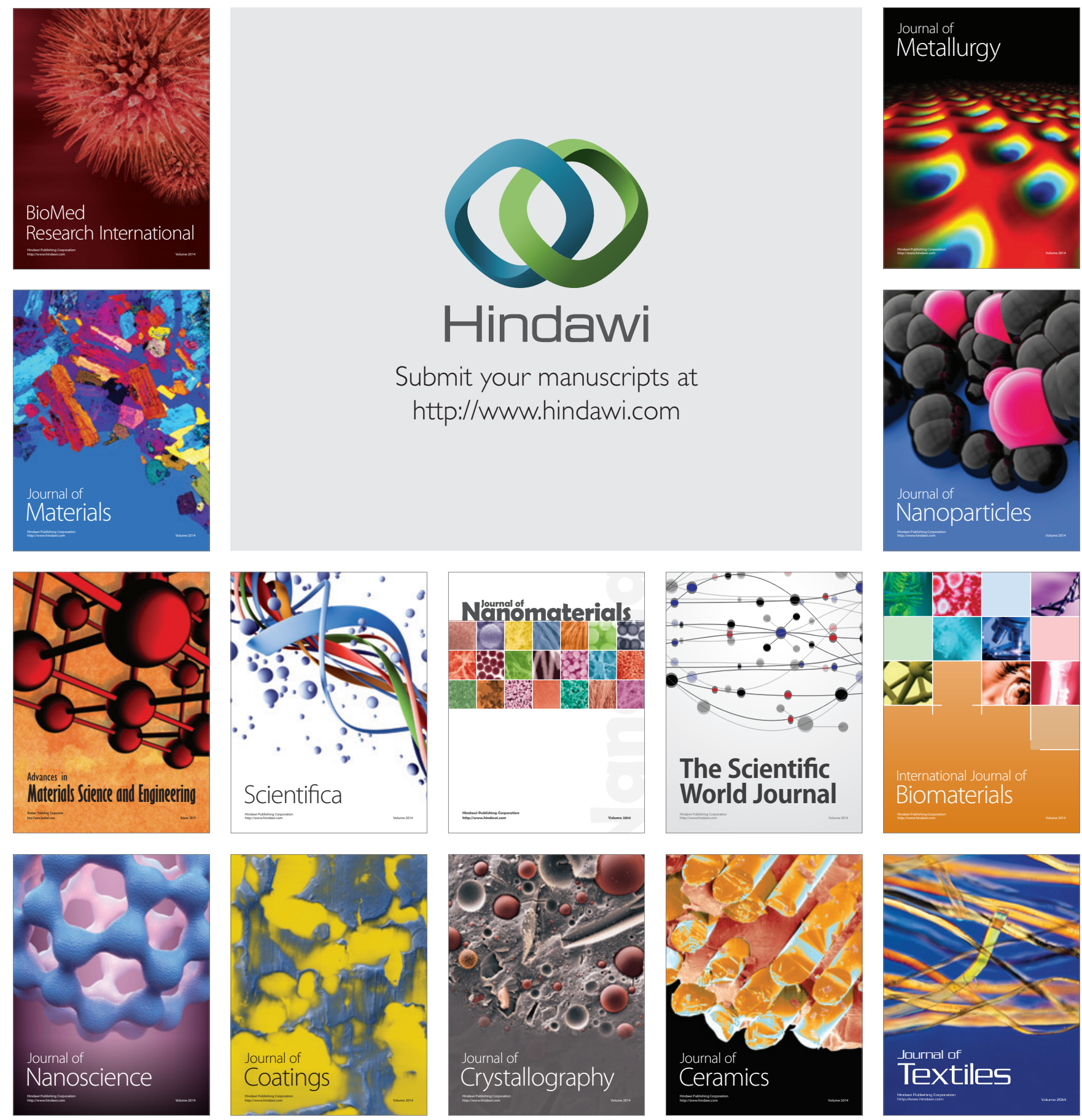